\title{
A SHORT PROOF OF THE GRIGORCHUK-COHEN COGROWTH THEOREM
}

\author{
RYSZARD SZWARC
}

(Communicated by J. Marshall Ash)

\begin{abstract}
Let $G$ be a group generated by $g_{1}, \ldots, g_{r}$. There are exactly $2 r(2 r-1)^{n-1}$ reduced words in $g_{1}, \ldots, g_{r}$ of length $n$. Part of them, say $\gamma_{n}$ represents identity element of $G$. Let $\gamma=\lim \sup \gamma_{n}^{1 / n}$. We give a short proof of the theorem of Grigorchuk and Cohen which states that $G$ is amenable if and only if $\gamma=2 r-12$. Moreover we derive some new properties of the generating function $\sum \gamma_{n} z^{n}$.
\end{abstract}

Let $G$ be a finitely generated discrete group. Consider $G$ as an epimorphic image $\pi: \mathbf{F}_{r} \rightarrow G$ of the free group $\mathbf{F}_{r}$ on $r$ generators. Thus $G$ is isomorphic to the quotient group $\mathbf{F}_{r} / N$ where $N=\operatorname{ker} \pi$ is a normal subgroup of $\mathbf{F}_{r}$. Once we fix a set of free generators in $\mathbf{F}_{r}$ we introduce $|x|$ the length of the word $x$ in $\mathbf{F}_{r}$ with respect to the generators and their inverses. Let $N_{n}=\{x \in N:|x|=n\}$, $\gamma_{n}=\operatorname{card} N_{n}$ and $\gamma=\lim \sup \gamma_{n}^{1 / n}$ which is called the growth exponent of $N$ is $\mathbf{F}_{r}$ with respect to the fixed set of free generators in $\mathbf{F}_{r}$. Because there are exactly $2 r(2 r-1)^{n-1}$ elements of length $n$ in $\mathbf{F}_{r}, \gamma \leq 2 r-1$. Grigorchuk [2] and Cohen [1] have shown that a group $G$ is amenable if and only if $\gamma$ attains maximal possible value, i.e. $\gamma=2 r-1$. We propose a new rather simple proof without any estimates which allows us to draw out new information on the behaviour of the generating function $N(z)=\sum \gamma_{n} z^{n}$.

As in [1] and [2] we will base our proof on a characterization of discrete amenable groups given by Kesten [3]. Any absolutely summable function $f$ on a group $G$ defines a translation invariant operator of $l^{2}(G)$ as $g \mapsto f * g$. The norm of corresponding map we denote as usual by $\|f\|_{\epsilon_{\text {red }}^{*}(G)}$.

Theorem 1 (Kesten [3]). Let $f$ be any selfadjoint summable positive function on $G$ whose support generates $G$. Then $G$ is amenable if and only if $\|f\|_{C_{\text {red }}^{*}(G)}=$ $\sum_{x \in G} f(x)$.

Received by the editors May 24, 1988.

1980 Mathematics Subject Classification (1985 Revision). Primary 43A07, 20F05; Secondary $22 \mathrm{D} 25$.

This work was written while the author held C.N.R. fellowship at the University of Rome "La Sapienza". 
As in [1] let $\chi_{n}, n=0,1,2, \ldots$ denote the characteristic function of the set of works of length $n$ in $\mathbf{F}_{r}$. Observe that the support of the function $\pi\left(\chi_{1}\right)$ generates $G$ by assumption. We are going to compute the norm $\left\|\pi\left(\chi_{1}\right)\right\|_{C_{\text {red }}^{*}(G)}$. Theorem 2 (Grigorchuk [2], Cohen [1]). Let $q=2 r-1$. then $\left\|\pi\left(\chi_{1}\right)\right\|_{C_{\text {red }}^{*}(G)}=$ $\gamma+q / \gamma$ only if $\gamma>1$ (or equivalently $\operatorname{ker} \pi$ is nontrivial).

Together with Kesten's theorem it gives immediately the following

Theorem 3 (Grigorchuk [2], Cohen [1]). $G$ is amenable if and only if $\gamma=2 r-1$. Proof of Theorem 2. The linear functional $C_{\text {red }}^{*}(G) \ni f \mapsto f(e)$ is the faithful trace $\operatorname{Tr}$ on $C_{\text {red }}^{*}(G)$ thus (see [6])

$$
\|f\|_{C_{\text {red }}^{*}(G)}=\lim _{n}\left(\operatorname{Tr} f^{* 2 n}\right)^{1 / 2 n}=\lim _{n}\left(\frac{\operatorname{Tr} f^{*}(2 n+2)}{\operatorname{Tr} f^{* 2 n}}\right)^{1 / 2} .
$$

Let $t$ be a positive number such that $0<t<q^{-1 / 2}$. If we let $\alpha(t)=q t+t^{-1}$ then by $[4$, proof of Theorem 3.1, p. 128] or by [5, Theorem 1] we have

$$
\left(\alpha(t) \delta_{e}-\chi_{1}\right)^{-1}(x)=\frac{t}{1-t^{2}} t^{|x|} \quad \text { in } C_{\mathrm{red}}^{*}\left(\mathbf{F}_{r}\right) .
$$

Thus using $(\alpha-x)^{-1}=\sum a^{-(n+1)} x^{n}$ we obtain

$$
\sum_{n=0}^{\infty} \alpha(t)^{-(n+1)} \chi_{1}{ }^{n}=\frac{t}{1-t^{2}} \sum_{n=0}^{\infty} t^{n} \chi_{n}
$$

Applying successively $\pi$ and then $\operatorname{Tr}$ to both sides and observing that $\operatorname{Tr} \pi\left(\chi_{n}\right)$ $=\gamma_{n}$ gives

$$
\sum_{n=0}^{\infty} \operatorname{Tr} \pi\left(\chi_{1}\right)^{{ }^{n}} \alpha(t)^{-(n+1)}=\frac{t}{1-t^{2}} \gamma_{n} t^{n} .
$$

(all this makes sense at least for values of $t$ small enough).

Now the point is that $(*)$ relates the radii of convergence: $r_{1}$ of the series $\sum \gamma_{n} t^{n}$ and $r_{2}$ of $\sum \operatorname{Tr} \pi\left(\chi_{1}\right)^{*} y^{n+1}$. Clearly we have $r_{1}=\gamma^{-1}$ and $r_{2}=\left\|\pi\left(\chi_{1}\right)\right\|_{C_{\text {red }}^{*}(G)}^{-1}$. Furthermore $\left\|\pi\left(\chi_{1}\right)\right\|_{C_{\text {red }}^{*}(G)} \geq\left\|\chi_{1}\right\|_{C_{\text {red }}^{*}\left(\mathbf{F}_{r}\right)}=2 q^{1 / 2}$ implies $r_{2} \leq \frac{1}{2} q^{-1 / 2}$ (see [5, Corollary 2], [1, Theorem 4]). Next using the fact that the function $y(t)=\alpha(t)^{-1}:\left[0, q^{-1 / 2}\right] \rightarrow\left[0, \frac{1}{2} q^{-1 / 2}\right]$ is increasing and the coefficients of the both series are nonnegative yields $y\left(r_{1}\right)=r_{2}$. But this gives the desired $\left\|\pi\left(\chi_{1}\right)\right\|_{C_{\text {red }}^{*}(G)}=\gamma+q / \gamma$.

Remarks. Let $\left\|\pi\left(\chi_{1}\right)\right\|_{C_{\text {red }}^{*}(G)}=\gamma+q / \gamma$. Then the function $z \mapsto \operatorname{Tr}\left[\alpha(z) \delta_{e}-\right.$ $\left.\pi\left(\chi_{1}\right)\right]^{-1}$ is analytic in the domain $\left\{z \in \mathbf{C}: \alpha(z) \notin\left[-\left(\gamma+q \gamma^{-1}\right), \gamma+q \gamma^{-1}\right]\right\}$. In particular it is analytic in $D=\left\{z \in \mathbf{C}:|z|<q^{-1 / 2}\right\} \backslash\left(\left[-q^{-1 / 2}, \frac{1}{\gamma}\right] \cup\left[\frac{1}{\gamma}, q^{-1 / 2}\right]\right)$. Hence by $(*)$ the function $z /\left(1-z^{2}\right) \sum \gamma_{n} z^{n}$ as well as $\sum \gamma_{n} z^{n}$ can be continued analytically to $D$. It means that $-\gamma^{-1}$ and $\gamma^{-1}$ are the only possible singular points on the circle of convergence of the power series $N(z)=\sum \gamma_{n} z^{n}$. 
Added in proof. After submission of the manuscript I was informed of the paper by W. Woess, Cogrowth of groups and simple random walks, Archiv der mathematic 41 (1983), 363-370, where the same arguments are used. In particular a formula similar to $(*)$ is proved there.

\section{REFERENCES}

1. J. M. Cohen, Cogrowth and amenability of discrete groups, J. Functional Analysis 48 (3) (1982), 301-309.

2. R. I. Grigorchuk, Symmetrical random walks on discrete groups, in "Multicomponent Random Systems" ed. R. L. Dobrushin, Ya. G. Sinai, pp. 132-152, Nauka, Moscow 1978 (English translation: Advances in Probability and Related Topics, Vol. 6, pp. 285-325, Marcel Dekker 1980.

3. H. Kesten, Full Banach mean values on countable groups, Math. Scand. 7 (1959), 149-156.

4. T. Pytlik, Radial functions on free groups and a decomposition of the regular representation into irreducible components, J. Reine Angew. Math. 326 (1981), 124-135.

5. R. Szwarc, An analytic series of irreducible representations of the free group, Ann. Inst. Fourier (Grenoble) 38 (1) (1988), 87-110.

6. S. Wagon, Elementary problems E 3226, Amer. Math. Monthly 94 (8) (1987), 786-787.

Institute of Mathematics, Wroclaw University, Pl. Grunwaldzki 2/4, PL-50-384 Wroclaw, Poland

Dipartimento di Matematica, instituto "Castelnuovo", Università "La Sapienza", Piazzale Aldo Moro 2, I-00185 Roma, Italy 